\title{
INTRODUCTION OF IMPERFECTIONS IN THE CUBIC MESH OF THE TRUSS-LIKE DISCRETE ELEMENT METHOD
}

\author{
Ignacio Iturrioz $^{1}$, Jorge Daniel Riera ${ }^{2}$ Letícia Fleck Fadel Miguel $^{1}$ \\ ${ }^{1}$ Department of Mechanical Engineering, UFRGS, Porto Alegre, Brazil<ignacio@mecanica.ufrgs.br> \\ ${ }^{2}$ Department of Civil Engineering, UFRGS, Porto Alegre, Brazil
}

\begin{abstract}
In the truss-like Discrete Element Method (DEM), masses are lumped at nodal points and interconnected by means of one-dimensional elements with arbitrary constitutive relations. In previous studies of non-homogeneous concrete cubic samples subjected to nominally uniaxial tension, it was verified that numerical predictions of fracture using DEM models are feasible and yield results that present good correlation with the experimental evidence so far available, including the prediction of size and strain rate effects. In the DEM approach, material failure under compression is assumed to occur by indirect tension. In previous simulations it was verified that the response is satisfactorily modeled up to the peak load, when a sudden collapse usually occurs, characteristic of fragile behavior. On the other hand, experimental stress vs. displacement curves observed in small specimens subjected to compression typically present a softening branch, in part due to sliding with friction of the fractured parts of the specimens. A second deficiency of DEM models with a perfectly cubic mesh is that the best correlations with experimental results are obtained with material parameters that differ in tension and compression. This paper examines another cause of the fragile behavior of DEM predictions of the response of concrete elements subjected to nominally uniaxial compression, which is due to the regularity of the perfect cubic mesh, which is unable to capture nonlinear stability effects in the material at a micro-scale. It is shown herein that the introduction of small perturbations of the DEM regular mesh significantly improves the predicting capability of the model and in addition allows adopting a unique set of material properties, which are independent of the applied loading.
\end{abstract}

\section{INTRODUCTION}

Krajcinovic (1996) classifies the methods proposed to predict the damage process in quasi-fragile materials in two large groups, those based on Continuum Mechanics, i.e. the classical approach, and the so-called Statistical Models approach. In the former, Plasticity Theories are extended to study the damage process, leading to procedures that encounter serious difficulties when dealing with quasi fragile materials, in which scale effects, anisotropic damage and associative behavior among defects are likely to occur. Examples of the Continuum Mechanics approach are the classical model due to Ottosen (1975) and the recent contribution of Crawford et al. (2012), who proposed a procedure implemented in the commercial finite element package $L S$-Dyna (Hallquist, 2007) to model damage in quasi-fragile materials. On the other hand, in the Statistical Models, the versatility of the finite element approach is lost, but in compensation problems such as localization, anisotropic damage evolution and associative effects between different parts of the specimen can be accounted for with relative facility. Examples of the second approach are provided by $\mathrm{Li}$ and Liu (2008), who review applications of discrete models consisting of particles in a mesh free distribution. This method was incorporated to the 2012 version of the commercial Finite element program Abaqus (2012), confirming the consolidation of statistical methods, as an appealing alternative to solve problems in which discontinuities appear during the damage process. Lattice Models, of which the present DEM formulation is a special case, belong to this group. Basically, the solid is modeled by means of an array of uniaxial elements, which interconnect nodal masses with two or three degrees of freedom. The stiffness of these elements can be determined from the mechanical properties of the anisotropic solid to be represented by the DEM. Schlangen (1995) and Rinaldi (2011) employ different versions of the Lattice approach. 
The Lattice DEM formulation used in this paper was proposed by Riera (1984) to determine the dynamic response of plates and shells under impact loading, when failure occurs primarily by shear or tension, which is generally the case in concrete structures. The constitutive criterion is based on Hillerborg's model (1971). Studies to predict the strength of large rock dowels subjected to shear were presented by Miguel et al. (2008). Rios and Riera (2004) apply DEM models to predict size effects in reinforced concrete beams. Others DEM applications in studies of non-homogeneous materials subjected to fracture were reported by Rocha et al. (1991), Dalguer et al. (2001), Kosteski et al. (2010) and Riera et al. (2011). DEM applications to the analysis of a NPP containment structures are described by Riera and Iturrioz (1998) and by Kostesky et al. (2011).

The behavior of fragile or quasi-fragile materials under simple tension is better known than under compression, which is more complex. In addition to Krajcinovic (1996), several authors studied this problem, proposing approaches such as the Sliding Crack Mechanism, which appears to capture the post peak part of the damage process (Nemat Nasser and Hori, 1982). Gross and Seelig (2006) describe the behavior of fragile materials subjected to compression: in a fist stage, a micro-fissures field is formed in the same direction of load application. Then, influenced by heterogeneities of the material, the fissures grow in inclined directions and mode II of fracture appears with subsequent sliding with friction, which happens after the peak load is reached.

This paper presents predictions of the response of prismatic concrete samples subjected to nominally static uniaxial compression obtained by simulation with the Discrete Element Method, assuming that rupture occurs only due to induced tensile strains in the material. Predictions of the peak compressive stress and of the rupture configuration compatible with experimental results presented by Ferrara and Gobbi (1995) are obtained by introducing imperfections in the cubic mesh geometry. However, the softening branch of the load vs. global displacement curves observed in laboratory experiments of concrete samples under uniaxial compression is not predicted correctly because it implies sliding with friction between fractured parts of the sample, capability that has not been yet implemented.

\section{THE DISCRETE ELEMENT METHOD IN FRACTURE PROBLEMS}

The Discrete Element Method employed in this paper is based on the representation of a solid by means of a cubic arrangement of elements able to carry only axial loads. The discrete elements representation of the orthotropic continuum was adopted to solve structural dynamics problems by means of explicit direct numerical integration of the equations of motion, assuming the mass lumped at the nodes. Each node has three degrees of freedom, corresponding to the nodal displacements in the three orthogonal coordinate directions. Figure 1 (a), (b) illustrates the basic bar arrangement used in this approach. The equations that relate the properties of the elements to the elastic constants of an isotropic medium are:

$$
\delta=\frac{9 v}{4-8 v}, \quad E A_{n}=E L^{2} \frac{(9+8 \delta)}{2(9+12 \delta)}, \quad E A_{d}=\frac{2 \sqrt{3}}{3} A_{n}
$$

in which $E$ and $v$ denote Young's modulus and Poisson's ratio, respectively, while $A_{n}$ and $A_{d}$ represent the areas of normal and diagonal elements. The resulting equations of motion may be written in the wellknown form:

$$
\mathbf{M} \overrightarrow{\tilde{x}}+\mathbf{C} \overrightarrow{\dot{x}}+\vec{F}_{r}(t)-\vec{P}(t)=0
$$

in which $\vec{x}$ represents the vector of generalized nodal displacements, $\boldsymbol{M}$ the diagonal mass matrix, $\boldsymbol{C}$ the damping matrix, also assumed diagonal, $\vec{F}_{r}(t)$ the vector of internal forces acting on the nodal masses and $\vec{P}(t)$ the vector of external forces. Obviously, if $\boldsymbol{M}$ and $\boldsymbol{C}$ are diagonal, Eq. (2) is not coupled. Then the explicit central finite differences scheme may be used to integrate Eq. (2) in the time domain. Since the nodal coordinates are updated at every time step, large displacements can be accounted for in a natural and efficient manner. 


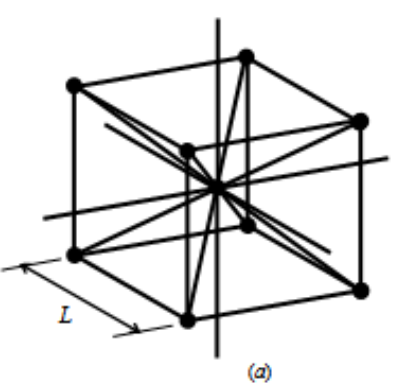

(a)

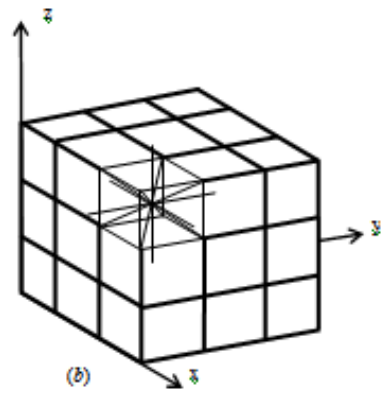

(b)

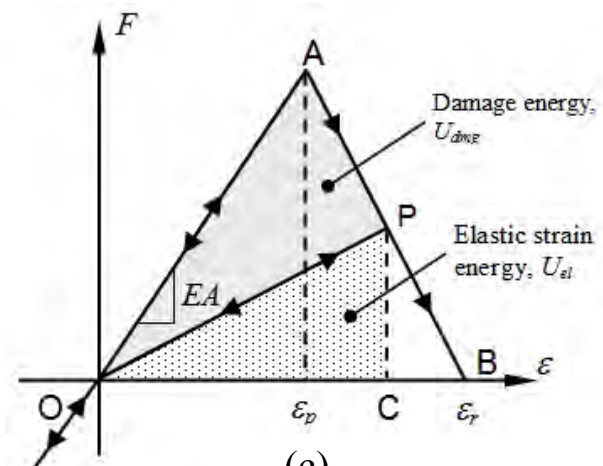

(c)

Figure 1: DEM discretization strategy: (a) basic cubic module, (b) generation of a prismatic body, (c) Bilinear constitutive law adopted for DEM uni-axial elements.

\section{Non-Linear Constitutive Model for Material Damage}

The softening law for quasi-fragile materials proposed by Hilleborg (1971) was adopted to handle the behavior of quasi-fragile materials by means of the triangular elemental constitutive relationship (ECR) for the DEM bars presented in Figure 1 (c) which allows accounting for the irreversible effects of crack nucleation and propagation. The area under the force $v s$. strain curve (the area of the triangle OAB) is related to the energy density necessary to fracture the area of influence of the element. Thus, for a given point $\mathrm{P}$ on the force $v s$. strain curve, the area of the triangle OPC quantifies the energy density dissipated by damage. Once the damage energy density equals the fracture energy, the element fails and loses its load carrying capacity. On the other hand, under compression the material is assumed linearly elastic. Thus, failure in compression is induced by indirect tension. Constitutive parameters and symbols shown in Figure 1 (c) are defined below: the element axial force $F$ depends on the axial strain $\varepsilon$. The area associated to each element is given by Eq. (6) for longitudinal and diagonal elements. An equivalent fracture area $A_{i}{ }^{*}$ of each element is defined in order to satisfy the condition that the energy dissipated by fracture of the continuum and by its discrete representation are equivalent. With this purpose, fracture of a cubic sample of dimensions $L \times L \times L$ is considered. The energy dissipated by fracture of a continuum cube due to a crack parallel to one of its faces is:

$$
\Gamma=G_{f} \Lambda=G_{f} L^{2}
$$

in which $\Lambda$ is the actual fractured area, i.e., $L^{2}$. On the other hand, the energy dissipated when a DEM module of dimensions $L \times L \times L$ fractures in two parts consists of the contributions of five longitudinal elements (four coincident with the module edges and an internal element) and four diagonal elements. Then, the energy dissipated by a DEM module can be written as (Kosteski et al., 2010):

$$
\Gamma_{\mathrm{DEM}}=G_{f}\left(40.25 c_{A}+c_{A}+4 c_{A}\left(\frac{2}{\sqrt{3}}\right)^{2}\right) L^{2}
$$

The first term between brackets accounts for the four edge elements, the second term for the internal longitudinal element, while the third term represents the contribution of the four diagonal elements. The coefficient $c_{A}$ is a scaling parameter used to establish the equivalence between $\Gamma$ and $\Gamma_{\text {DEM }}$. Thus:

$$
G_{f} L^{2}=G_{f}\left(\frac{22}{3} c_{A}\right) L^{2}
$$


from eq.(5) it follows that $c_{A}=3 / 22$. Finally, the equivalent fracture areas of longitudinal and diagonal elements are:

$$
A_{l}{ }^{*}=(3 / 22) L^{2}, \quad A_{d}{ }^{*}=(4 / 22) L^{2}
$$

These values apply as long as there is a single large crack in the element. The critical failure strain $\left(\varepsilon_{p}\right)$ is defined as the largest strain attained by the element before damage initiation (point $\mathrm{A}$ in Figure 1c). The relationship between $\varepsilon_{p}$ and the specific fracture energy $G_{f}$ is given in terms of Linear Elastic Fracture Mechanics as:

$$
\varepsilon_{p}=R_{f} \sqrt{\frac{G_{f}}{E\left(1-v^{2}\right)}}
$$

in which $R_{f}$ is the so-called failure factor, which may account for the presence of an intrinsic defect of size a. $R_{f}$ may be expressed in terms of $a$ as:

$$
R_{f}=\frac{1}{Y \sqrt{a}}
$$

in which $Y$ is a dimensionless parameter that depends on both the specimen and crack geometry. The element loses its load carrying capacity when the limit strain $\varepsilon_{r}$ is reached (Point B in Figure 1c). This value must satisfy the condition that, upon failure of the element, the dissipated energy density equals the product of the element fracture area $A_{i}{ }^{*}$ times the specific fracture energy $G_{f}$, divided by the element length. Hence:

$$
\int_{0}^{\varepsilon_{r}} F(\varepsilon) d \varepsilon=\frac{G_{f} A_{i}^{*}}{L_{i}}=\frac{K_{r} \varepsilon_{p}^{2} E A_{i}}{2}
$$

in which the sub index $i$ is replaced by $l$ or $d$ depending on whether the element under consideration is longitudinal or diagonal. The coefficient $K_{r}$ is a function of the material properties and the element length $L_{i}$ :

$$
K_{r}=\left(\frac{G_{f}}{E \varepsilon_{p}^{2}}\right)\left(\frac{A_{i}^{*}}{A_{i}}\right)\left(\frac{2}{L_{i}}\right)
$$

In order to guarantee the stability of the algorithm, the condition $K_{r} \geq 1$ must be satisfied. In this sense, it is interesting to define the critical element length:

$$
\begin{gathered}
L_{c r}=2\left(\frac{G_{f}}{E \varepsilon_{p}^{2}}\right)\left(\frac{A_{i}^{*}}{A_{i}}\right) \\
\left(A_{l}^{*} / A_{l}\right)=(3 / 22) / \phi, \quad\left(A_{d}^{*} / A_{d}\right)=(\sqrt{3} / 11) /(\delta \phi)
\end{gathered}
$$

In the special case of an isotropic continuum with $v=0.25$, the value of the coefficients above are $\delta=1.125$ and $\phi=0.4$, which lead to $\left(A_{l}{ }^{*} / A_{l}\right) \approx\left(A_{d}{ }^{*} / A_{d}\right) \approx 0.34$. Thus, for practical purposes, a single value of the critical length can be used for longitudinal and diagonal elements. Therefore, the stability condition is expressed by equation (13) and the limit strain by equation (14):

$$
\begin{gathered}
K_{r}=\frac{L_{c r}}{L_{i}} \geq 1 \Rightarrow L_{i} \leq L_{c r} \\
\varepsilon_{r}=K_{r} \varepsilon_{p}
\end{gathered}
$$




\section{The random distribution of material parameters in the DEM environment}

Miguel et al (2010) and Iturrioz et al (2009) modeled the random properties of the material defining the toughness $G_{f}$ as a random field with a Type III (Weibull) extreme value distribution, given by Eq. (15):

$$
F\left(G_{f}\right)=1-\exp \left[-\left(G_{f} / \beta\right)^{\gamma}\right]
$$

in which $\beta$ and $\gamma$ are the scale and shape parameters, respectively. The mean value $(\mu)$ and the standard deviation (s) of $G_{f}$ are given by:

$$
\begin{gathered}
\mu=\beta[\Gamma(1+1 / \gamma)] \\
s=\beta\left[\Gamma(1+2 / \gamma)-\Gamma^{2}(1+1 / \gamma)\right]^{1 / 2}
\end{gathered}
$$

in which $\Gamma(x)=\int_{0}^{\infty} t^{x-1} e^{-t} d t$ denotes the Gamma function. In order to simulate pseudo random values of $G_{f}$, the following expression was used:

$$
G_{f}=\beta[-\ln (1-u)]^{1 / \gamma}
$$

in which $u$ is a random number with uniform probability distribution in the interval $(0,1)$. Routines for generating samples of $u$ are widely available. In earlier applications of the DEM, taking the size of the elements $(L)$ equal to the correlation length of the random field of the material property of interest, say $l_{c}$, allowed assuming that simulated values were uncorrelated, thus simplifying the computational scheme. This is however an important restriction and procedures to generate DEM meshes that are independent from the correlation length of the random field were used by Miguel et al. (2008) and Puglia et al. (2010).

\section{PERTURBATIONS OF THE DEM CUBIC ARRANGEMENT}

In the present formulation of the Discrete Element Method, solids are represented by means of a cubic arrangement of elements able to carry only axial loads, interconnected at nodal masses with three degrees of freedom. The initial elastic stiffness of the interconnecting elements is determined, for the cubic and other arrangements, in terms of the local elastic properties of an orthotropic solid, which may be non-homogeneous, by means of available sets of equations. The introduction of small perturbations of the cubic arrangement, generated by small initial displacements of nodal points, should also result in small changes in the stiffness of the elements, which will tend to zero as the initial nodal displacements vanish. Hence, it is herein assumed that the stiffness coefficients of the DEM model remain unaltered by small perturbations of the mesh.

Moreover, the linear response of the model should remain unaltered within the range of interest, whose upper limit will be established later. Basically, it is assumed that the nodes in the perturbed model are displaced from their position in a perfect cubic arrangement, defined by nodal coordinates $\left(x_{n}, y_{n}, z_{n}\right)$, as indicated below:

$$
\left(x_{n}+r_{x} L, y_{n}+r_{y} L, z_{n}+r_{z} L\right)
$$

in which $r_{x}, r_{y}$ and $r_{z}$ are random numbers with a normal distribution with zero mean and coefficient of variation $C_{p}$. $L$ denotes the length of the longitudinal elements in the cubic cell. The $C_{p}$ value that best fits the experimental evidence was determined by numerical experimentation. 


\section{ANALYSIS OF CUBIC SAMPLES SUBJECTED TO UNIAXIAL COMPRESSION}

The response of $150 \mathrm{~mm}$ cubic concrete samples tested by Ferrara and Gobbi (1995) under uniaxial compression was numerically determined using DEM models. The samples had no lateral restrictions at the upper and lower faces. Material properties were $\mu_{G f}=150 \mathrm{~N} / \mathrm{m}, C V_{G f}=50 \%$, $\rho=2400 \mathrm{~kg} / \mathrm{m}^{3}, R_{f c}=4, L=0.0075 \mathrm{~m}$. The response of simulated samples was determined for a perfect mesh of the DEM arrangement and then after introducing perturbations in the mesh with $C_{p}$ equal to 1 , 2.5 and $5 \%$, while maintaining the distribution of material properties. The response of samples under uniaxial compression predicted by the four models is presented in Fig. 2 (top left), while Fig. 2 (top right) shows the predictions for uniaxial tension of the same simulated samples. On the other hand, Fig. 2 (bottom left) illustrates the influence of the variability of the material toughness $G_{f}$ defined by $C V_{G f}$. This factor plays an important role in tensile loading, when fracture Mode I is the dominant phenomenon, as shown by the stress-displacement curves of Fig. 2 (bottom right). A $C V_{G f}=0.5$ value had been adopted in previous studies of samples subjected to nominally uniaxial tension (Iturrioz et al., 2009 and Riera et al., 2011) and was maintained herein. Coefficient $C_{p}=2.5 \%$ leads to predictions that best fit the experimental results on cubic samples under compression, while exerting a marginal influence on the response of samples subjected to uniaxial tension, whose behavior is dominated by $C V_{G f}$. Fig. 2 (top left) also shows Ferrara and Gobbi (1995) experimental results. Note that the linear response (small stress or displacement) is the same for the various DEM imperfect meshes (characterized by different values of $C_{p}$ ). As expected, the numerically predictions present a close correlation up the peak value of the stressdisplacement curves. Afterwards the numerical response falls more abruptly. The experimental curves, on the other hand, present a softening branch produced by a complex mechanism of splitting and then sliding among fractured parts of the sample. It is quite clear that the softening branch of the experimental curves does not characterize any material behavior, but rather the behavior of the sample structural system.
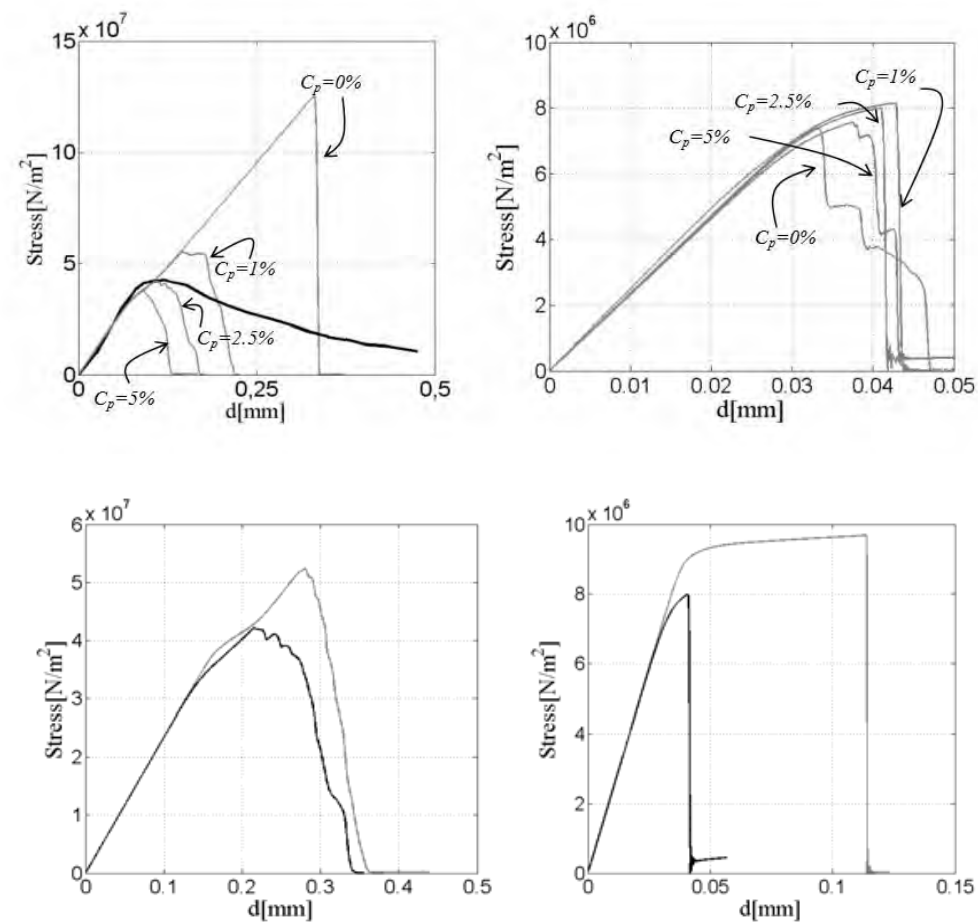

Figure 2: Influence of DEM mesh on the response of $150 \mathrm{~mm}$ cubes under compression (top left) and tension (top right) (experimental results in thick lines). DEM predictions of the response of samples under uniaxial compression (bottom left) and uniaxial tension (bottom right) for $C V_{G f}=50 \%$ (thick lines) and $C V_{G f}=0 \%$ (thin lines). 


\section{ANALYSIS OF PRISMATIC SAMPLES SUBJECTED TO UNIAXIAL COMPRESSION}

Concrete prismatic samples fixed at the lower face and subjected to a monotonically increasing uniformly distributed compression displacement on the upper face were analyzed next. The response of each sample up to failure was determined through numerical simulation. Two sets with different boundary conditions were analyzed. First (Case A), the samples were analyzed without restrictions in $x$ and $y$ directions on the nodes on the upper and lower faces. Next, (Case B), the same samples were analyzed with restrictions in both $x$ and $y$ directions. Samples with $0.15 \times 0.15 \mathrm{~m}$ cross sectional area and 0.15 (short specimens AS or BS), 0.30 (medium specimens AM or BM) and $0.45 \mathrm{~m}$ (long specimens AL or BL) lengths were analyzed. The size $L$ of DEM elements was $0.015 \mathrm{~m}$ in all cases. Hence, the three sample sizes contain $10 \times 10 \times 10 ; 10 \times 10 \times 20$ and $10 \times 10 \times 30$ modules, respectively. The material properties of concrete are: Young's modulus, $E=3.5 \mathrm{E} 10 \mathrm{~N} / \mathrm{m}^{2}$; mass density, $\rho=2400 \mathrm{~kg} / \mathrm{m}^{3}$ and Poisson's ratio, $v=$ 0.25 . In the ensuing simulations, the specific fracture energy, $G_{f}$ is assumed a random field with mean $90 \mathrm{~N} / \mathrm{m}$ and a coefficient of variation equal to $50 \%$. The critical strain $\varepsilon_{p}$ is assumed equal to $3.9 \mathrm{E}-5$ and the $\mathrm{CV}$ of the imperfections set equal to $C_{p}=0.5 \%$. Nodal points on the upper face of the specimens were subjected to a controlled (compression) displacement that increases smoothly in time, inducing a nominally uniform compression in the specimen. Six simulations were performed for each sample size. By introducing small imperfections in the cubic mesh, satisfactory values for the DEM predictions both in tension and compression, are obtained.

In Fig. 3 the results show the model capability to predict the samples behavior up to the peak load, i.e. their compressive strength, mean strain at failure configuration. Note that none of the models presents a clear scale effect for the peak stress and, as expected, fail to correctly account for the postfailure behavior, affected by shear sliding with friction. Moreover, the variability of the compressive strength observed in the three sample sizes predicted by the numerical models (Simulated CV between 10 and $16 \%$ ) is larger than typically expected for concrete (between 5 and $10 \%$ ).

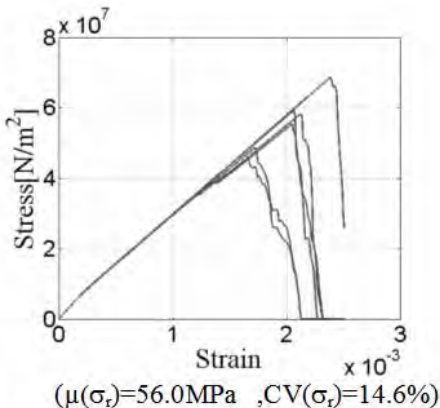

(a)

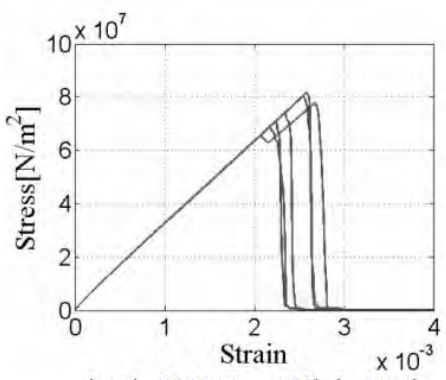

$\left(\mu\left(\sigma_{\mathrm{r}}\right)=75.8 \mathrm{MPa}, \mathrm{CV}\left(\sigma_{\mathrm{r}}\right)=6.7 \%\right)$

(d)

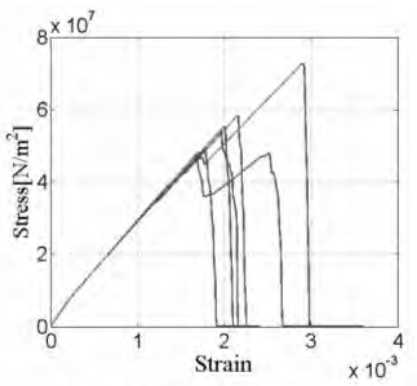

$\left(\mu\left(\sigma_{\mathrm{r}}\right)=56.1 \mathrm{MPa}, \mathrm{CV}\left(\sigma_{\mathrm{r}}\right)=16.2 \%\right)$

(b)

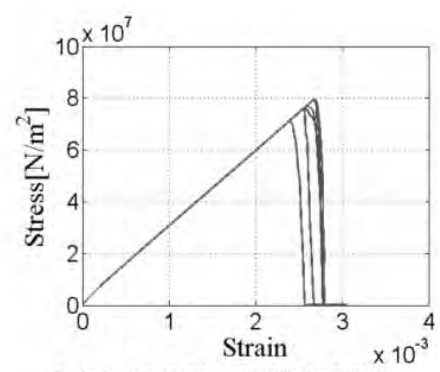

$\left(\mu\left(\sigma_{\mathrm{r}}\right)=76.9 \mathrm{MPa}, \mathrm{CV}\left(\sigma_{\mathrm{r}}\right)=3.9 \%\right)$

(e)

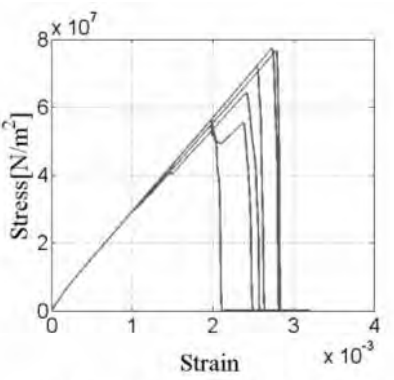

$\left(\mu\left(\sigma_{\mathrm{r}}\right)=67.1 \mathrm{MPa} \quad, \mathrm{CV}\left(\sigma_{\mathrm{r}}\right)=14.5 \%\right)$

(c)

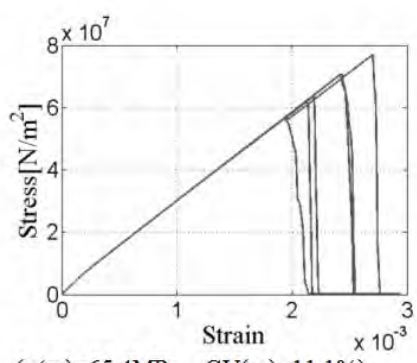

$\left(\mu\left(\sigma_{\mathrm{r}}\right)=65.4 \mathrm{MPa}, \mathrm{CV}\left(\sigma_{\mathrm{r}}\right)=11.1 \%\right)$

(f)

Figure 3: Mean normal stress on the lower face $v s$. mean strain for 6 simulations. Diagrams (a), (b), (c) correspond to three different slenderness ratios without mesh imperfections, while diagrams (d) (e) (f) presents the stress-strain curves obtained including the mesh imperfections. 
Typical final configurations obtained with DEM are presented in Figures 4 and 5 . The appearance of inclined fractures at approximately $45^{\circ}$, along which sliding with friction occurs at a later stage, is clearly seen in Case B in agreement with experimental evidence. However, the incipient failure configurations predicted by the models with mesh imperfections do not show the appearance of the vertical "columns", visible in the models with a perfect mesh and not typical of experimental results.

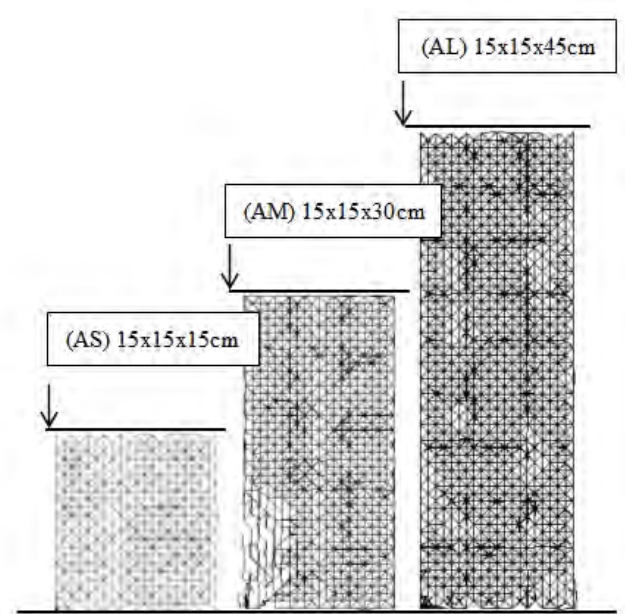

(a) Case $\mathrm{A}$

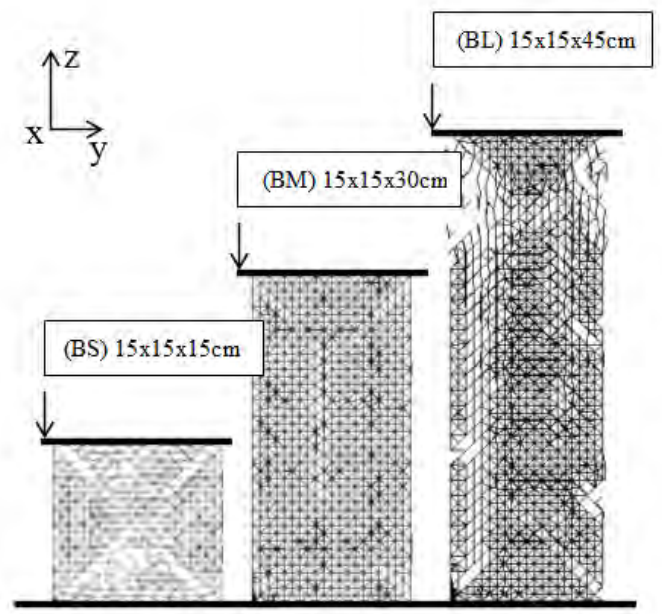

(b) Case B

Figure 4: Rupture configurations of concrete samples with mesh imperfections subjected to uniaxial compression (controlled displacements). Only damage at central slices of the samples is shown. Case A without lateral restrictions, Case B with lateral restrictions at end platens. (Fractured bars are not visualized in the configurations).

\section{(a) Case A}

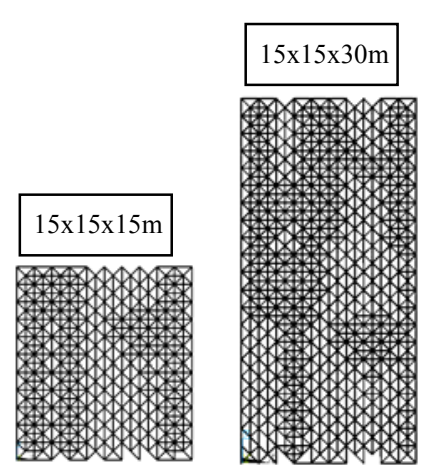

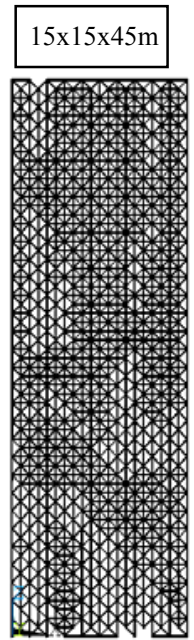

(b) Case B

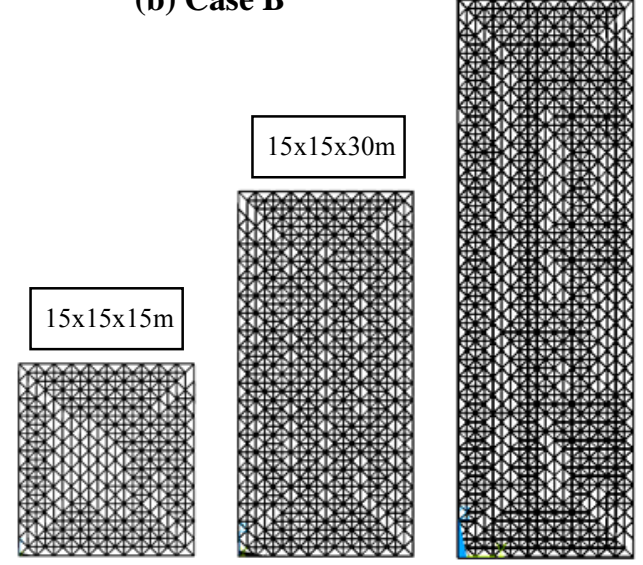

Figure 5: Rupture configuration of concrete samples without mesh imperfections subjected to uniaxial compression (controlled displacements). Only damage at central slices of the samples is shown. Case A without lateral restrictions, Case B with lateral restrictions at end platens. (Fractured bars are not visualized in the configurations). 
$22^{\text {nd }}$ Conference on Structural Mechanics in Reactor Technology

San Francisco, California, USA - August 18-23, 2013

Division IX (include assigned division number from I to X)

\section{CONCLUSIONS}

The so-called Discrete Element Method (DEM) has become an appealing option for the analysis of structural systems subjected to fracture and fragmentation, in which case it constitutes a natural approach to the problem, in contrast to methods based on Continuum Mechanics such as the Finite Element Method (FEM), which remain, on the other hand, as the preferred and more robust alternative in the case of linear or weakly nonlinear problems. The authors proposed in the early 80's and extensively tested in the last two decades a truss-like DEM model based on a cubic mesh arrangement, investigating its performance in static and dynamic, linear and nonlinear problems, including the verification of critical loads, fracture and failure configurations. It was also verified that truss-like DEM models were able to predict size and strain rate effects in concrete and other semi-fragile materials.

The authors contend that introducing small perturbations in the cubic mesh, a single set of material parameters can be used for cases in which failure occurs due to uniaxial tension or compression. Since both are extreme situations, it may be expected that the conclusion applies as well for other intermediate loading conditions.

In samples that fail by tension, the numerically determined fracture patterns as well as the softening branches of the stress-strain or load-displacement curves present a close correlation with experimental results. In the case of elements subjected to nominally uniaxial compression, on the other hand, while the carrying capacity and the fracture patterns are correctly predicted by the present DEM formulation with the imperfect cubic mesh, the predicted behavior is more fragile than in physical samples, that is, in small elements a sudden decrease of the simulated stress-strain curve occurs shortly after the peak load is reached. This is not he case in physical samples, due to the occurrence of sliding with friction along fracture surfaces originated earlier in the loading process, which are not yet considered in the analysis.

The results presented suggest that the truss-like DEM formulation with the imperfect mesh described in the paper can be resorted to in order to numerically predict the load carrying capacity of concrete or other quasi fragile elements subjected to arbitrary quasi static and dynamic excitations.

Acknowledgements. The authors acknowledge the support of CNPq and CAPES (Brazil).

\section{REFERENCES}

Abaqus/Explicit, (2011) Version 6.x, Dassault Systemes Americas Corp, Waltham, MA.

Crawford, J. E. Crawford, Y. Wu, J.M Magallanes \& S. Lan, Chap 1 in the Advances in protective Structures Research- Hao \&li (eds). 2012 Taylor \& francis Group, London ISBN 978-0-415-64337-5

Dalguer, L.A., Irikura, K., Riera, J.D., Chiu H.C. (2001), "The importance of the dynamic source effects on strong ground motion during the 1999 Chi-Chi, Taiwan, earthquake: Brief interpretation of the damage distribution on buildings". Bull. Seismol. Soc. Am., Vol. 91, pp. 1112-1127.

Ferrara, G. and Gobbi, M. E. (1995) Strain Softening of Concrete Under Compression. Report to RILEM Committee 148-SSC, ENEL-CRIS Laboratory, Milano, Italy.

Gross D. and Seelig T. (2006). "Fracture Mecanics with and Introduction to Micromechanics", Mechnaics Engineering Series. Ed. Ling F. F., Springer. Pp319. doi 10.1007/b22134.

Hallquist, J.O., (2007) LS-DYNA - Keyword User's Manual, Version 971, Livermore Software Technology Corporation (LSTC), California, USA.

Hillerborg, A. (1971): “A model for fracture analysis”, Cod. LUTVDG/TVBM 300-51-8, 1971.

Iturrioz, I., Miguel, L.F.F., Riera, J.D. (2009): "Dynamic Fracture Analysis of Concrete or Rock Plates by Means of the Discrete Element Method", LAJSS, Vol. 6, pp. 229-245. 
$22^{\text {nd }}$ Conference on Structural Mechanics in Reactor Technology

San Francisco, California, USA - August 18-23, 2013

Division IX (include assigned division number from I to X)

Iturrioz, I.; Riera, J.D.; Miguel, L.F.F. and Kosteski, L.E. (2011): "Scale effects in quasi-fragile materials subjected to compression", Div. I, Paper \#54, $21^{\text {th }}$ International Conference on Structural Mechanics in Reactor Technology (SMiRT 21), Delhi, India.

Kosteski, L.E., Riera, J.D., Iturrioz, I. (2010), "Consideration of Scale Effects and Stress Localization in Response Determination Using the DEM", MECOM - CILAMCE 2010, November 2010, Buenos Aires, Argentina. Mecánica Computacional, Vol XXIX, pp. 2785-2801.

Kosteski L.E., Riera J.D., Iturrioz I. (2011): "Ultimate load analysis of Barcom test model made with the TrussLike Discrete Element Method", Int. Conf. and Structural Mechanics in Reactor Technology, SMiRT 21, Trans. Vol. I., New Delhi, India.

Kosteski, L.; Iturrioz, I. and Riera, J.D. (2012): "Global and local constitutive laws assessment in the truss-like Discrete Elelemnt Method applications", (in portuguese), XXXV Jornadas Sul Americanas de Engenharia Estrutural, Rio de Janeiro, Set. 2012 (in CD).

Krajcinovic D., (1996) "Damage Mechanics". Elsevier, Amsterdam.

Liu G. B., Liu M. B. (2007) Smoothed Particled Hydrodinamics a Meshfree Particle methods, World Scientific Publ.

Miguel, L.F.F., Riera, J.D., Iturrioz, I. (2008), "Influence of size on the constitutive equations of concrete or rock dowels", Int J Numer Anal Meth Geomech, Vol. 32, No. 15, pp. 1857-188, 2008. DOI: 10.1002/nag.699.

Miguel, L.F.F., Iturrioz, I., Riera, J.D. (2010), "Size Effects and Mesh Independence in Dynamic Fracture Analysis of Brittle Materials", Computer Modeling in Engineering \& Sciences, Vol. 56, pp. 1-16.

Munjiza, A (2009) Special issue on the discrete element method: aspects of recent developments in computational mechanics of discontinua, Engineering Computations, Vol. 26 Iss: 6, pp.

Nemat-Nasser, S. and Horii, H., (1982), Compression-Induced Nonplanar Crack Extension with Application to Splitting, Exfoliation and Rock Burst, J. Geophys. Res., Vol. 87, pp. 6805-6821.

Ottosen N. S., (1975). "Failure and Elasticity of Concrete" Riso_M1801.

Puglia, B.V., Iturrioz, I, Riera, J.D., Kosteski, L.(2010), "Random field generation of the material properties in the truss-like discrete element method”, Mec. Comp., Cilamce-Mecom 2010, Vol. XXIX, pp. 6793-6807.

Riera, J.D. (1984): "Local effects in impact problems on concrete structures". Proceedings, Conference on Structural Analysis and Design of Nuclear Power Plants. Oct. 1984, Porto Alegre, RS, Brasil, Vol. 3, CDU 264.04:621.311.2:621.039.

Riera, J.D., Iturrioz, I. (1998):“Discrete elements model for evaluating impact and impulsive response of reinforced concrete plates and shells subjected to impulsive loading”. Nuclear Engineering and Design, Vol. 179, pp. $135-144$

Riera, J.D., Miguel, L.F.F., Iturrioz, I., (2011), "Strength of Brittle Materials under High Strain Rates in DEM Simulations", Computer Modeling in Engineering \& Sciences, Vol. 82, pp. 113-136.

Rinaldi, A. (2011) “Advances In Statistical Damage Mechanics: New Modelling Strategies”, In: Damage Mechanics and Micromechanics of Localized Fracture Phenomena in Inelastic Solids, Voyiadjis G. (Ed.), CISM Course Series, Springer.

Rios, R.D, Riera, J.D. (2004): "Size effects in the analysis of reinforced concrete structures", Engineering Structures, Elsevier, Vol. 26, pp. 1115-1125, 2004.

Rocha, M.M., Riera, J.D., Krutzik N.J. (1991): "Extension of a model that aptly describes fracture of plain concrete to the impact analysis of reinforced concrete", Int. Conf. and Structural Mechanics in Reactor Technology, SMiRT 11, Trans. Vol. J., Tokyo, Japan.

Schlangen, E. and van Mier, J.G.M., Crack propagation in sandstone: Combined experimental and numerical approach. Rock Mech. and Rock Eng., 1995, 28(2), pp.93-110. 